\title{
Nongrowing Preferential Attachment Random Graphs
}

\author{
Tomas Hruz and Ueli Peter
}

Abstract. We consider an edge rewiring process that is widely used to model the dynamics of scale-free weblike networks. This process uses preferential attachment and operates on sparse multigraphs with $n$ vertices and $m$ edges. We prove that its mixing time is optimal and develop a framework that simplifies the calculation of graph properties in the steady state. The applicability of this framework is demonstrated by calculating the degree distribution, the number of self-loops, and the threshold for the appearance of the giant component.

\section{Introduction}

The study of complex networks has attracted considerable attention over the past ten years. Recently, there have appeared signals suggesting that a new phase in network development, importance, and proliferation is on the way. Some networks like the Internet and the World Wide Web have become mission-critical. Even some military operations are partially supported through the Internet, not to mention news reports and information from geographical regions under crisis. The importance of complex networks has led to deeper research showing that the behavior and the growth of such networks are far from being purely random. They often follow certain topological and structural patterns discovered in the

(C) Taylor \& Francis Group, LLC

ISSN: I542-795I print 
theory of scale-free networks and small worlds [Barabási and Albert 99, Watts and Strogatz 98].

During the past decade an interplay between statistical analysis of real-world networks and a formal study of stochastic models has turned out to be promising (see [Cami and Deo 07] for a survey on methods used for the analysis of such models). The models are designed to simulate dynamic change in a network and to produce properties observed in real-world networks. A formal analysis is important in order to verify the quality of the model by comparing it with empirically collected data [Mitzenmacher 06]. Unfortunately, the well-understood random graph models $G_{n, p}$ and $G_{n, m}$ do not produce the features that are observed in real-world networks, nor are they designed to simulate the dynamics of those networks. The research community is therefore challenged to design and analyze better models.

The most-discussed property of complex networks is the scale-free degree distribution [Barabási and Albert 99]. The first model to create graphs with a scale-free degree distribution was the Barabási-Albert model, which was rigorously analyzed in [Bollobás et al. 01]. That model adds a vertex at every time step and connects it to the existing graph by choosing a vertex with preferential attachment. This is believed to model the growing phase of a network quite well. However, it is known [Park et al. 05] that several complex networks reach a state of saturation in which they no longer grow but continue to evolve. Unfortunately, there are almost no rigorous results known for models of nongrowing networks. In this paper we make a first step toward analyzing such a model.

A widely accepted stochastic process [Dorogovtsev and Mendes 03, Evans and Plato 07, Hruz et al. 08] that uses preferential attachment and considers multigraphs with a stable number of vertices and edges is defined as follows. At every time step, one of the $m$ edges in the multigraph is selected uniformly at random and one of its half-edges is rewired to a new vertex, which is selected at random from $n$ vertices with probability proportional to a preference function $f(d(v)):\{1, \ldots, 2 m\} \rightarrow \mathbb{R}$, where $d(v)$ is the degree of vertex $v$. If there is more than one graph, we write $d_{G}(v)$ for the degree of vertex $v$ in graph $G$. The symbol $\langle f\rangle$ stands for the average value $\left(\sum_{v} f(d(v))\right) / n$ of $f$. This process is called SESP (simple edge-selection process) and has the following formal definition.

Process I.I. (SESP.) The following steps are repeated at each time unit:

1. An edge $e_{i}$ is selected uniformly at random.

2. An end vertex $v_{i}$ of $e_{i}$ is selected uniformly at random. 
3. A vertex $v_{l}$ is selected at random with probability $f\left(d\left(v_{l}\right)\right) / n\langle f\rangle$.

4. The edge $e_{i}$ is rewired from $v_{i}$ to $v_{l}$.

In [Evans and Plato 07], the authors solved a mean-field equation for the degree distribution of Process 1.1. We complement the understanding of this model with a rigorous study of the graph properties in the steady state of the process. We believe that SESP represents one of the simplest possible processes that is nevertheless principally different from classical random graphs. This claim is supported by the fact that if the preference function is set to a constant, then every multigraph appears with equal probability in the stationary distribution that is equivalent to a multigraph version of $G_{n, m}$. The next natural step, which is tackled in this paper, is to consider linear preference functions.

\section{I.I. SESP Markov Chains}

The parameters of Process 1.1 are the number of vertices $n$, the number of edges $m$, the preference function $f$, and the input graph $G_{0}$. To reformulate the model as a Markov chain, we define the state space $\Omega$ that contains all multigraphs with $n$ labeled vertices and $2 m$ labeled half-edges. We split each edge $e$ in the middle and denote its two half-edges by $e^{1}$ and $e^{2}$. Let the vertices and half-edges be labeled $v_{1}, \ldots, v_{n}$ respectively $e_{1}^{1}, e_{1}^{2}, \ldots, e_{m}^{1}, e_{m}^{2}$. Then a labeled multigraph $x \in \Omega$ is defined as $\left(H\left(v_{1}\right), \ldots, H\left(v_{n}\right)\right)$, where $H\left(v_{i}\right)$ denotes the set of half-edges at vertex $v_{i}$. The transition probabilities between two states in $\Omega$ explicitly follow from Process 1.1. The result of steps 1 and 2 in Process 1.1 is that every halfedge is selected with probability $1 /(2 m)$. Therefore, we will use the following equivalent definition of the process for the remainder of this paper.

Process I.2. (SESP Markov chain.) The following steps are repeated at each time unit:

1. A half-edge $e_{i}^{j}$ (where $1 \leq i \leq m$ and $j \in\{0,1\}$ ) is selected uniformly at random from all half-edges.

2. A vertex $v_{l}$ is selected at random with probability $f\left(d\left(v_{l}\right)\right) / n\langle f\rangle$.

3. The half-edge $e_{i}^{j}$ is rewired to $v_{l}$.

Note that for $x, y \in \Omega$ there is a nonzero transition probability if and only if there exists at most one half-edge $e_{i}^{1}$ that is at different vertices in $x\left(v_{p}\right)$ and $y$ 
$\left(v_{q}\right)$. Then a transition from $x$ to $y$ happens only if $e_{i}^{1}$ is selected in step 1 and $v_{q}$ is selected in step 2 of Process 1.2. Thus,

$$
P(x, y)=\frac{1}{2 m} \frac{f\left(d_{x}\left(v_{q}\right)\right)}{n\langle f\rangle} \text { and } P(y, x)=\frac{1}{2 m} \frac{f\left(d_{y}\left(v_{p}\right)\right)}{n\langle f\rangle},
$$

where $d_{s}(v)$ denotes the degree of vertex $v$ in state $s$. The Markov chain is irreducible if and only if $f(k)>0$ for all $k \in\{0, \ldots, 2 m\}$, because then every state can be reached from every other state in at most $2 m$ transitions. It is furthermore aperiodic (and therefore ergodic) because a transition from state $G \in \Omega$ back into itself has positive probability for all states. It follows from the fundamental Markov chain theorem (see, for example, [Levin et al. 09, Theorem 4.16]) that the chain will converge into its unique stationary distribution $\pi$.

All our results in this article are for linear preference functions $f(k)=a k+b$, where $a \geq 0$ and $b>0$ are constants such that $a+b=1$. In [Evans and Plato 07], the authors explained that selection of a vertex of degree $k$ with probability

$$
p_{p} \frac{k}{2 m}+p_{r} \frac{1}{n}
$$

where $p_{p}$ and $p_{r}$ are probabilities that add up to 1 , is the most general form of linear preferential attachment. Because of

$$
\frac{f(k)}{n\langle f\rangle}=\frac{a k+b}{n(a \bar{k}+b)}=\frac{a}{a+\frac{b}{\bar{k}}} \frac{k}{2 m}+\frac{b}{a \bar{k}+b} \frac{1}{n}=p_{p} \frac{k}{2 m}+p_{r} \frac{1}{n}
$$

for

$$
a:=\frac{p_{p}}{\bar{k} p_{r}+p_{p}} \quad \text { and } \quad b:=\frac{\bar{k} p_{r}}{\bar{k} p_{r}+p_{p}},
$$

our choice of the preference function is equivalent. We require that $b$ be strictly larger than zero, because otherwise, the Markov process is not irreducible and will converge to a particular set of states. When $b=0$, the analysis of the process is simple, since the graph condenses to a state in which all edges are connected to a single vertex. Complex networks are usually sparse, and we therefore assume that the average degree $\bar{k}=2 \mathrm{~m} / n$ is constant.

In this paper we study graph properties in the stationary distribution of the SESP Markov chain and can therefore neglect the initial graph $G_{0}$. We justify this proceeding by showing that the process reaches the stationary distribution rapidly. Note that the authors of [Evans and Plato 07] chose a different approach. They derived the dynamic of the degree distribution over time by solving a meanfield equation for the number of vertices of degree $k$ at time $t$.

Let $G_{n, a, \bar{k}}$ denote the random graph model in which multigraphs on $n$ vertices and $m=n \bar{k} / 2$ edges are distributed according to the stationary distribution of 
the SESP Markov chain with preference function $f(k)=a k+b$ (where $b:=1-a$ and $0 \leq a<1)$. Note that for such a function, the average is

$$
\langle f\rangle=a \bar{k}+b=a(\bar{k}-1)+1
$$

We use $G \sim G_{n, a, \bar{k}}$ to denote that graph $G$ is drawn at random with distribution $G_{n, a, \bar{k}}$. For a graph property $\mathcal{P}$ we say that $G_{n, a, \bar{k}}$ has $\mathcal{P}$ asymptotically almost surely (a.a.s.) or with high probability (whp) whenever the probability that $G \sim$ $G_{n, a, \bar{k}}$ has $\mathcal{P}$ is $1-o(1)$, where $n$ is the relevant parameter for all asymptotic notations unless otherwise stated.

A natural question that arises when we examine a graph property is this: For what parameters $a$ and $\bar{k}$ does $G_{n, a, \bar{k}}$ exhibit the given property as $n$ tends to infinity?

\subsection{Main Results}

Our first result shows that the Markov chain converges rapidly, which legitimates our approach of looking only at the stationary distribution.

Theorem I.3. The mixing time $t_{\mathrm{mix}}(\epsilon)$ of SESP is bounded by

$$
t_{\text {mix }}(\epsilon) \leq \log \left(n \bar{k} \epsilon^{-1}\right) n c_{1},
$$

where $c_{1}:=\bar{k}(a(\bar{k}-1)+1) /(1-a)$.

This upper bound on the mixing time is asymptotically optimal, since every half-edge needs to be rewired at least once to have a nonzero probability that two processes with initial graphs of distance $2 m$ are in the same state. It is well known (see, for example, the coupon collector problem in [Levin et al. 09, Section 2.2 ]) that $O(m \log m)$ steps are needed to have a high probability for the event that all $2 m$ half-edges have been selected at least once.

Inequalities that give a measure for the concentration of a quantity around its expectation appeared helpful in the study of random graph models. A 1-Lipschitz function $f: \Omega \rightarrow \mathbb{R}$ has the property that for all $x, y \in \Omega$,

$$
\frac{|f(x)-f(y)|}{h(x, y)} \leq 1
$$

where $h(x, y)$ is the shortest-path metric in the Markov chain.

We observe that many important graph measures such as the number of isolated vertices, the chromatic number, and the number of self-loops are Lipschitz functions. In this work we develop the following concentration inequality for 1-Lipschitz functions. 
Theorem I.4. For all 1-Lipschitz functions $f: \Omega \rightarrow \mathbb{R}$ and all $u>0$, if $G \sim G_{n, a, \bar{k}}$, then

$$
\operatorname{Pr}[|f(G)-\mathbb{E}[f(G)]| \geq u] \leq 2 e^{-u^{2} / n c_{1}}
$$

where

$$
c_{1}:=\frac{\bar{k}(a(\bar{k}-1)+1)}{1-a} .
$$

The degree distribution is an important topic in complex network research. In this paper we use the random variable $N(k)$ to denote the number of vertices of degree $k$ in $G_{n, a, \bar{k}}$. In the following theorem we derive the expected number of vertices of a given degree in $G \sim G_{n, a, \bar{k}}$.

Theorem 1.5. For every $\varepsilon>0, G \sim G_{n, a, \bar{k}}$ has a.a.s. no vertex of degree larger than $k_{\max }:=\left(2+\varepsilon / \log c_{2}\right) \log m$, and for all $0 \leq k \leq k_{\max }$, the expected number of vertices of degree $k$ has the following asymptotic form:

$$
\mathbb{E}[N(k)]=\Theta\left(n \cdot \frac{1}{(1+b / a \bar{k})^{k}} \cdot k^{-1+b / a}\right) .
$$

One consequence of Theorem 1.4 is that the degree distribution is concentrated around its expectation.

Corollary 1.6. For $0 \leq k \leq 2 m$ and $G \sim G_{n, a, \bar{k}}$,

$$
\operatorname{Pr}[|N(k)-\mathbb{E}[N(k)]| \geq \sqrt{n \log n}] \leq 2 n^{-1 / 4 c_{1}},
$$

where

$$
c_{1}:=\frac{\bar{k}(a(\bar{k}-1)+1)}{1-a}
$$

The configuration model for random graphs is the state-of-the-art method for the examination of random graphs with a specific degree distribution. For a degree sequence $d_{1}, \ldots, d_{n}$, where $d_{i}$ denotes the degree of $v_{i}$, we write $G \sim \operatorname{Conf}\left(d_{1}, \ldots, d_{n}\right)$ if $G$ is chosen at random out of all multigraphs with probability defined by the configuration model. Theorem 1.7 states that in $G_{n, a, \bar{k}}$, all multigraphs of a given degree sequence have the same probability as in the configuration model. 
Theorem I.7. Let $G_{\mathrm{SESP}} \sim G_{n, a, \bar{k}}$, and for an arbitrary degree sequence $d_{1}, \ldots, d_{n}$ such that $\sum d_{i}=n \bar{k}$, let $G_{\mathrm{Conf}} \sim \operatorname{Conf}\left(d_{1}, \ldots, d_{n}\right)$. Then for all multigraphs $G$ with degree sequence $d_{1}, \ldots, d_{n}$,

$$
\operatorname{Pr}\left[G_{\mathrm{SESP}}=G \mid d_{G_{\mathrm{SESP}}}\left(v_{1}\right)=d_{1}, \ldots, d_{G_{\mathrm{SESP}}}\left(v_{n}\right)=d_{n}\right]=\operatorname{Pr}\left[G_{\mathrm{Conf}}=G\right] .
$$

In [Molloy and Reed 95, Molloy and Reed 00], the authors derived the threshold for the appearance of the giant component in the configuration model for a large class of random graphs. In Section 3.2, we apply the framework provided by the previous theorems to show that $G_{n, a, \bar{k}}$ is in that class. This results in a precise formulation of the threshold as a function of $a$ and $\bar{k}$ in Lemma 4.2. We believe that there exists a simple formulation of the threshold, namely that there is a giant component if $\bar{k}>(1-a)$ and that all components are small if $\bar{k}<(1-a)$, as formulated in Conjecture 4.3. We prove the conjecture for two important special cases of the preference function.

In Section 2, we prove Theorems 1.3 and 1.4. In Section 3, we derive Theorem 1.5 and Corollary 1.6. Then in Section 3.1, we prove Theorem 1.7 by looking at symmetries in the transition diagram of the Markov chain. All these results are used in Section 4 to derive the number of self-loops and the threshold for the appearance of the giant component. At the end we compare our results to previous work and draw a conclusion.

\section{Mixing Time of SESP}

We use the following standard consequence (see, for example, [Dyer et al. 00, Theorem 2.1]) of the coupling lemma [Aldous 83] to prove an upper bound on the mixing time of the SESP Markov chain.

Lemma 2.I. Let $\left(X_{t}, Y_{t}\right)$ be a coupling of a Markov chain and let $\rho$ be any integer-valued metric defined on $\Omega \times \Omega$. Suppose that there exists $\beta \leq 1$ such that $\mathbb{E}\left[\rho\left(X_{t+1}, Y_{t+1}\right)\right] \leq \beta \rho\left(X_{t}, Y_{t}\right)$ for all $t$ and all $\left(X_{t}, Y_{t}\right) \in \Omega \times \Omega$. Let $D$ be the maximum value that $\rho$ achieves on $\Omega \times \Omega$. If $\beta<1$, then the mixing time $t_{\text {mix }}(\epsilon)$ of the Markov chain satisfies

$$
t_{\text {mix }}(\epsilon) \leq \frac{\log \left(D \epsilon^{-1}\right)}{(1-\beta)} .
$$

We now use the above tool to prove the optimal mixing time of the SESP Markov chain. 
Proof of Theorem 1.3. The transition probabilities (1.1) imply that $P(x, y)>0$ if and only if $P(y, x)>0$, and the Markov chain therefore has an underlying undirected graph induced on the states $\Omega$, where $x$ and $y$ are connected by an edge if and only if $P(x, y)>0$. Let $h(x, y)$ denote the length of the shortest path between the two states $x$ and $y$ in this undirected graph.

The central idea of the proof is to define a coupling $\left(X, X^{\prime}\right) \in \Omega \times \Omega$ of the Markov chain such that we can use the shortest path distance $h\left(x, x^{\prime}\right)$ (which is clearly a metric) and apply Lemma 2.1 . Let $X$ be an exact copy of the SESP Markov chain with the transition probabilities described in (1.1). Our goal is to design $X^{\prime}$ in such a way that the expected distance between $X$ and $X^{\prime}$ decreases after every transition. Note that for all $x, x^{\prime} \in \Omega$, the distance $h\left(x, x^{\prime}\right)$ counts exactly the number of half-edges that are in $x$ connected to vertices different from those in $x^{\prime}$. We are therefore tempted to define $X^{\prime}$ such that it selects the same half-edge as $X$ and rewires it to the same vertex as in $X$. But this would not be a valid coupling, since the marginal transition probabilities of $X^{\prime}$ would be different from those in the original SESP Markov chain. We therefore have to change this idea slightly to create a formally correct coupling of the Markov chain.

To describe the transition probabilities of $X^{\prime}$ we let $d(v)$ denote the degree of vertex $v$ in $X$, and $d^{\prime}(v)$ the degree of $v$ in $X^{\prime}$. Assume without loss of generality that in $X$ we have selected half-edge $e_{i}^{1}$ and rewired it to vertex $v_{p}$. Then if $f\left(d\left(v_{p}\right)\right) \geq f\left(d^{\prime}\left(v_{p}\right)\right)$, we rewire $e_{i}^{1}$ in $X^{\prime}$ to vertex $v_{p}$ with probability

$$
\frac{f\left(d^{\prime}\left(v_{p}\right)\right)}{f\left(d\left(v_{p}\right)\right)},
$$

and with probability

$$
\left(1-\frac{f\left(d^{\prime}\left(v_{p}\right)\right)}{f\left(d\left(v_{p}\right)\right)}\right) \frac{f\left(d^{\prime}\left(v_{q}\right)\right)-f\left(d\left(v_{q}\right)\right)}{\Delta}
$$

to

$$
v_{q} \in U^{\prime}:=\left\{v \mid f\left(d^{\prime}(v)\right)>f(d(v))\right\},
$$

where

$$
\Delta:=\sum_{v_{l} \in U^{\prime}} f\left(d^{\prime}\left(v_{l}\right)\right)-f\left(d\left(v_{l}\right)\right) .
$$

We furthermore define

$$
U:=\left\{v \mid f(d(v))>f\left(d^{\prime}(v)\right)\right\}
$$


and observe that

$$
\Delta:=\sum_{v_{l} \in U^{\prime}} f\left(d^{\prime}\left(v_{l}\right)\right)-f\left(d\left(v_{l}\right)\right)=\sum_{v_{l} \in U} f\left(d\left(v_{l}\right)\right)-f\left(d^{\prime}\left(v_{l}\right)\right)
$$

for linear preference functions. If $f\left(d\left(v_{p}\right)\right)<f\left(d^{\prime}\left(v_{p}\right)\right)$, we rewire $e_{i}^{1}$ to $v_{p}$.

It remains to show that the marginal transition probabilities of $X^{\prime}$ are equal to the transition probabilities of SESP. The marginal edge-selection probability in $X^{\prime}$ is clearly $1 / 2 m$ for every half-edge. The vertex-selection probability for a vertex $v_{i}$ with $f\left(d^{\prime}\left(v_{i}\right)\right) \leq f(d(v))$ is

$$
\frac{f\left(d\left(v_{i}\right)\right)}{n\langle f\rangle} \frac{f\left(d^{\prime}\left(v_{i}\right)\right)}{f\left(d\left(v_{i}\right)\right)}=\frac{f\left(d^{\prime}\left(v_{i}\right)\right)}{n\langle f\rangle} .
$$

For a vertex with $f\left(d^{\prime}\left(v_{i}\right)\right)>f(d(v))$, the vertex selection probability is

$$
\begin{aligned}
& \frac{f\left(d\left(v_{i}\right)\right)}{n\langle f\rangle}+\sum_{v_{l} \in U} \frac{f\left(d\left(v_{l}\right)\right)}{n\langle f\rangle}\left(1-\frac{f\left(d^{\prime}\left(v_{l}\right)\right)}{f\left(d\left(v_{l}\right)\right)}\right) \frac{f\left(d^{\prime}\left(v_{i}\right)\right)-f\left(d\left(v_{i}\right)\right)}{\Delta} \\
& =\frac{f\left(d\left(v_{i}\right)\right)}{n\langle f\rangle}+\frac{f\left(d^{\prime}\left(v_{i}\right)\right)-f\left(d\left(v_{i}\right)\right)}{n\langle f\rangle \Delta} \sum_{v_{l} \in U} f\left(d\left(v_{l}\right)\right)-f\left(d^{\prime}\left(v_{l}\right)\right)=\frac{f\left(d^{\prime}\left(v_{i}\right)\right)}{n\langle f\rangle} .
\end{aligned}
$$

We observe that the distance between the states $x$ and $x^{\prime}$ of the coupling $\left(X, X^{\prime}\right)$ decreases if a half-edge that is on a vertex in $X$ different from that in $X^{\prime}$ is selected and rewired to the same vertex in $X$ and $X^{\prime}$. Similarly, the distance increases if a half-edge that is on the same vertex in both states is selected and rewired to different vertices. Therefore, we can calculate the expectation of $h\left(X_{t+1}, X_{t+1}^{\prime}\right)$ as a function of $h\left(X_{t}, X_{t}^{\prime}\right)$ :

$$
\begin{aligned}
\mathbb{E}[ & \left.h\left(X_{t+1}, X_{t+1}^{\prime}\right)\right] \\
= & h\left(X_{t}, X_{t}^{\prime}\right)-\frac{h\left(X_{t}, X_{t}^{\prime}\right)}{2 m}\left(1-\sum_{v_{l} \in U} \frac{f\left(d\left(v_{l}\right)\right)}{n\langle f\rangle}\left(1-\frac{f\left(d^{\prime}\left(v_{l}\right)\right)}{f\left(d\left(v_{l}\right)\right)}\right)\right) \\
& +\left(1-\frac{h\left(X_{t}, X_{t}^{\prime}\right)}{2 m}\right) \sum_{v_{l} \in U} \frac{f\left(d\left(v_{l}\right)\right)}{n\langle f\rangle}\left(1-\frac{f\left(d^{\prime}\left(v_{l}\right)\right)}{f\left(d\left(v_{l}\right)\right)}\right) \\
= & h\left(X_{t}, X_{t}^{\prime}\right)-\frac{h\left(X_{t}, X_{t}^{\prime}\right)}{2 m}+\frac{1}{n\langle f\rangle} \sum_{v_{l} \in U} f\left(d\left(v_{l}\right)\right)-f\left(d^{\prime}\left(v_{l}\right)\right) \\
= & h\left(X_{t}, X_{t}^{\prime}\right)-\frac{h\left(X_{t}, X_{t}^{\prime}\right)}{2 m}+\frac{a}{n\langle f\rangle} \sum_{v_{l} \in U} d\left(v_{l}\right)-d^{\prime}\left(v_{l}\right) \\
\leq & h\left(X_{t}, X_{t}^{\prime}\right)-\frac{h\left(X_{t}, X_{t}^{\prime}\right)}{2 m}+\frac{a \cdot h\left(X_{t}, X_{t}^{\prime}\right)}{n\langle f\rangle} \\
= & h\left(X_{t}, X_{t}^{\prime}\right)\left(1-\frac{(a 2 m+n b)-a 2 m}{2 m n\langle f\rangle}\right)=h\left(X_{t}, X_{t}^{\prime}\right)\left(1-\frac{b}{n \bar{k}\langle f\rangle}\right) .
\end{aligned}
$$


There are $2 m$ half-edges that can be connected to different vertices in $x$ and $y$, which means that the distance between two states is always at most $D:=2 \mathrm{~m}$. We can therefore finish the proof by applying Lemma 2.1 and (1.2) to the coupling $\left(X, X^{\prime}\right)$ that satisfies $(2.1)$.

The following lemma was proven as [Luczak 08, Corollary 4.4].

Lemma 2.2. Suppose that there are a constant $0<\beta<1$ and two copies $\left(X, X^{\prime}\right)$ of a Markov chain with Wasserstein distance

$$
\operatorname{dist}_{W}\left(X_{1}, X_{1}^{\prime}\right) \leq \alpha
$$

for all $x_{0}, x_{0}^{\prime} \in \Omega$ with $h\left(x_{0}, x_{0}^{\prime}\right)=1$. Then if the Markov chain has stationary distribution $\pi$, it follows for all $u>0$ and every 1-Lipschitz function $f$ that

$$
\operatorname{Pr}(|f(X)-\mathbb{E}[f(X)]| \geq u) \leq 2 e^{-u^{2}\left(1-\beta^{2}\right) / 2 \beta^{2}},
$$

where $X$ is a random variable with distribution $\pi$.

Proof of Theorem I.4. It follows from the Kantorovich-Rubinstein theorem (see [Luczak 08] or for a more general discussion [Dudley 02]) that the Wasserstein distance at time 1 of two SESP Markov chains $Y_{t}$ and $Y_{t}^{\prime}$ with starting states $y_{0}$ and $y_{0}^{\prime}$ is bounded by

$$
\operatorname{dist}_{W}\left(Y_{1}, Y_{1}^{\prime}\right) \leq \mathbb{E}\left[h\left(X_{1}, X_{1}^{\prime}\right) \mid x_{0}=y_{0}, x_{0}^{\prime}=y_{0}^{\prime}\right],
$$

where $\left(X, X^{\prime}\right)$ is an arbitrary coupling of the Markov chain. Hence we can use the coupling defined above and conclude that for $x_{0}, x_{0}^{\prime}$ with $h\left(x_{0}, x_{0}^{\prime}\right)=1$, the Wasserstein distance is bounded by

$$
\operatorname{dist}_{W}\left(X_{1}, X_{1}^{\prime}\right) \leq\left(1-\frac{b}{n \bar{k}\langle f\rangle}\right) .
$$

Considering Lemma 2.2, this proves the theorem.

Note that many graph properties such as the number of isolated vertices, the number of self-loops, and the maximum degree are 1-Lipschitz functions. For some properties we derive the same bound by applying Theorem 1.7 and Azuma's inequality. However, this result is helpful only for functions $f$ for which $\mathbb{E}[f]=\Omega(\sqrt{n \log n})$, because otherwise, by Markov's inequality we already get a stronger bound. 


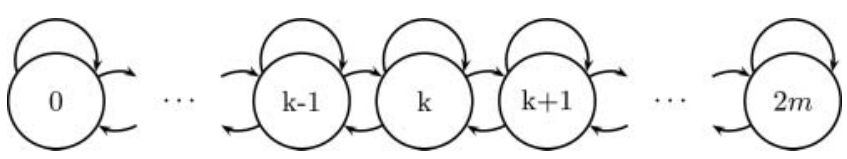

Figure I. Transition diagram of the degree Markov chain.

\section{The Degree Sequence}

In order to analyze the degree sequence in the stationary distribution, we calculate the probability $P(k)$ that an arbitrary vertex is of degree $k$. Then we show that the number of vertices of degree $k$ is sufficiently concentrated around its expectation $\mathbb{E}[N(k)]=n \cdot P(k)$. In one step of Process 1.2, a particular vertex $v$ gains a half-edge with probability $f(d(v)) /(n\langle f\rangle)$ and loses a half-edge with probability $d(v) /(2 m)$. For a linear preference function, the average value $\langle f\rangle=a \bar{k}+b$ is a constant that does not depend on the degree distribution $P(k)$. We therefore observe that the degree of any vertex $v$ at time $t+1$ depends only on the degree of $v$ at time $t$. Thus, the random variable that measures the degree of a vertex $v$ at time $t$ is an ergodic Markov chain that has the unique stationary distribution $P(k)$ for $0 \leq k \leq 2 m$. We will refer to this chain as the degree Markov chain (DMC). The states of the DMC are the possible degrees that the vertex can take $\left(\Omega_{\mathrm{DMC}}:=\{0,1, \ldots, 2 m\}\right)$. Note that SESP consist of $n$ instances of the DMC, which are all controlled by a single source of randomness.

The probability for a transition from state $x=k$ to state $y=k+1$ is

$$
P(x, y)=\left(1-\frac{k}{2 m}\right) \frac{f(k)}{n\langle f\rangle},
$$

since this transition requires the event that no half-edge of the vertex be removed and the event that the vertex be selected for rewiring. The remaining transition probabilities result from analogical reasoning. Table 1 lists all transition probabilities of the DMC.

We now use the DMC to prove Theorem 1.5.

Proof of Theorem 1.5. The DMC is a birth-and-death chain (see [Levin et al. 09, Section 2.5]) and therefore reversible. Hence we can calculate $P(k)$ from $P(0)$ by

$$
\begin{aligned}
P(k) & =\frac{P(k-1, k)}{P(k, k-1)} P(k-1)=\left(\prod_{i=0}^{k-1} \frac{P(i, i+1)}{P(i+1, i)}\right) \cdot P(0) \\
& =\frac{\prod_{i=0}^{k-1}(2 m-i) f(i)}{\prod_{i=1}^{k} i(n\langle f\rangle-f(i))} P(0) .
\end{aligned}
$$




\begin{tabular}{cccc}
\hline$x$ & $y$ & $P(x, y)$ & $P(y, x)$ \\
\hline$k$ & $k+1$ & $\left(1-\frac{k}{2 m}\right) \frac{f(k)}{n\langle f\rangle}$ & $\frac{k+1}{2 m}\left(1-\frac{f(k+1)}{n\langle f\rangle}\right)$ \\
$k$ & $k-1$ & $\frac{k}{2 m}\left(1-\frac{f(k)}{n\langle f\rangle}\right)$ & $\left(1-\frac{k-1}{2 m}\right) \frac{f(k-1)}{n\langle f\rangle}$ \\
$k$ & $k$ & $\left(1-\frac{k}{2 m}\right)\left(1-\frac{f(k)}{n\langle f\rangle}\right)+\frac{k}{2 m} \frac{f(k)}{n\langle f\rangle}$ \\
\hline
\end{tabular}

Table I. Transition probabilities for the degree Markov chain (DMC).

The normalization condition gives us

$$
P(0)=1 / \sum_{k=0}^{2 m} \prod_{i=1}^{k} \frac{f(i-1) \cdot(2 m-i+1)}{i \cdot(n\langle f\rangle-f(i))} .
$$

Hence for $0 \leq k \leq 2 m$,

$$
P(k)=\prod_{i=1}^{k} \frac{f(i-1) \cdot(2 m-i+1)}{i \cdot(n\langle f\rangle-f(i))} / \sum_{j=0}^{2 m} \prod_{i=1}^{j} \frac{f(i-1) \cdot(2 m-i+1)}{i \cdot(n\langle f\rangle-f(i))} .
$$

For $a=0$ we observe by a straightforward calculation that $G_{n, a, \bar{k}}$ is the binomial random graph for which

$$
P(k)=\left(\begin{array}{c}
2 m \\
k
\end{array}\right)\left(\frac{1}{n}\right)^{k}\left(\frac{n-1}{n}\right)^{2 m-k} .
$$

For $a>0$ we can rewrite the numerator of (3.2) using the gamma function:

$$
\begin{aligned}
& \prod_{i=1}^{k} \frac{f(i-1) \cdot(2 m-i+1)}{i \cdot(n\langle f\rangle-f(i))} \\
& \quad=\frac{\prod_{i=0}^{k-1}(a i+b)}{k !} \frac{\prod_{i=2 m-k+1}^{2 m} i}{\prod_{i=2 m-k}^{2 m-1}(a i+b(n-1))} \\
& \quad=\frac{a^{k} \prod_{i=0}^{k-1}\left(i+\frac{b}{a}\right)}{k !} \frac{\Gamma(2 m+1)}{\Gamma(2 m-k+1)} \frac{1}{a^{k} \prod_{i=2 m-k}^{2 m-1}\left(i+\frac{b}{a}(n-1)\right)} \\
& \quad=\frac{\Gamma\left(k+\frac{b}{a}\right)}{\Gamma\left(\frac{b}{a}\right) \Gamma(k+1)} \frac{\Gamma(2 m+1)}{\Gamma(2 m-k+1)} \frac{\Gamma\left(2 m-k+\frac{b}{a}(n-1)\right)}{\Gamma\left(2 m+\frac{b}{a}(n-1)\right)} .
\end{aligned}
$$




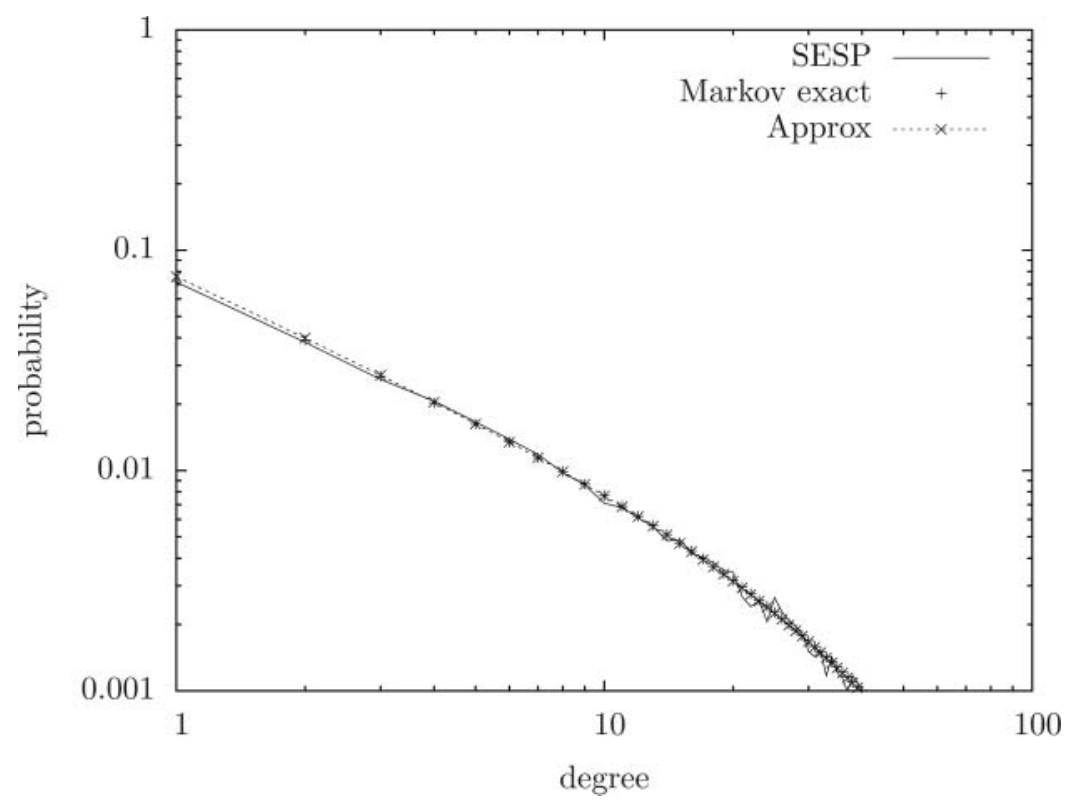

Figure 2. This illustration shows a plot of the exact stationary distribution of the Markov chain (3.2) against the approximation (3.6) and the degree distribution of a network after $10^{7}$ simulation steps of SESP. All data are for the preference function $f(k)=0.9 k+0.1$ and a network of size $n=1000$ and $m=2000$.

By applying Stirling's approximation of the gamma function (see Lemma 6.1) we can show that

$$
\frac{\Gamma\left(k+\frac{b}{a}\right)}{\Gamma\left(\frac{b}{a}\right) \Gamma(k+1)} \frac{\Gamma(2 m+1)}{\Gamma(2 m-k+1)} \frac{\Gamma\left(2 m-k+\frac{b}{a}(n-1)\right)}{\Gamma\left(2 m+\frac{b}{a}(n-1)\right)}=O\left(c_{2}^{-k} \cdot k^{-1+\frac{b}{a}}\right),
$$

where $c_{2}:=1+b / a \bar{k}$. Note that Lemma 6.1 states that for $0 \leq k \leq\left(\frac{2+\varepsilon}{\log c_{2}}\right) \log m$, the asymptotic upper bound is tight.

We use (3.4) to simplify the denominator of (3.2). There is some constant $c$ such that

$$
\sum_{j=0}^{2 m} \prod_{i=1}^{j} \frac{f(i-1) \cdot(2 m-i+1)}{i \cdot(n\langle f\rangle-f(i))}=\sum_{k=0}^{2 m} O\left(\left(1+\frac{b}{a \bar{k}}\right)^{-k}\right) \leq c \cdot \sum_{k=0}^{\infty}\left(1+\frac{b}{a \bar{k}}\right)^{-k} .
$$

Since this infinite sum converges, we conclude that

$$
P(k)=O\left(c_{2}^{-k} \cdot k^{-1+b / a}\right) .
$$


The exponential cutoff $\left(c_{2}^{-k}\right)$ of the degree distribution already indicates that the probability for vertices of large degree is small. We now show that for $\varepsilon>0$, there are a.a.s. no vertices of degree larger than $k_{\max }:=\left(\frac{2+\varepsilon}{\log c_{2}}\right) \log m$. By union bound and (3.5), the probability that there is a vertex of degree at least $k_{\max }$ is at most

$$
n \sum_{i=k_{\max }}^{2 m} P\left(k_{\max }\right) \leq 2 m \cdot n /\left(1+\frac{b}{a \bar{k}}\right)^{k_{\max }}=o(1) .
$$

Therefore, in the stationary distribution of SESP the maximum degree of a graph is asymptotically almost surely at most $\left(\frac{2+\varepsilon}{\log c_{2}}\right) \log m$, and $P(k)$ simplifies for all $k \leq\left(\frac{2+\varepsilon}{\log c_{2}}\right) \log m$ to

$$
P(k)=\Theta\left(c_{2}^{-k} \cdot k^{-1+b / a}\right) .
$$

The number of vertices of degree $k$ divided by two is a function from the state space of the Markov chain to the real numbers. The function is 1-Lipschitz because in one step of SESP, the number of vertices of degree $k$ increases or decreases by at most two, and therefore

$$
\forall_{x, x^{\prime} \in \Omega}: \frac{\left|N_{x}(k)-N_{x^{\prime}}(k)\right|}{2 d\left(x, x^{\prime}\right)} \leq 1 .
$$

Corollary 1.6 is a simple consequence of the above observation and Theorem 1.4. Note that we use $N_{x}(k)$ for the number of vertices of degree $k$ in graph $x$, which is slightly different from our usual notation.

\section{I. Relation to the Configuration Model}

The configuration model (see [Bollobás 80, Janson et al. 00]) is the standard method for studying random regular graphs and random graphs of a given degree sequence. For a degree sequence $d\left(v_{1}\right), d\left(v_{2}\right), \ldots, d\left(v_{n}\right)$, the first step is to construct the $n$ vertices containing the $2 m$ labeled half-edges. Then a configuration (a pairing of the $2 m$ half-edges) is chosen uniformly at random from all possible configurations. The multigraph that corresponds to the chosen configuration can be constructed by inserting an edge between every pair of half-edges. There are

$$
(2 m-1) ! !=(2 m-1) \cdot(2 m-3) \cdots 3 \cdot 1=\frac{(2 m) !}{2^{m} m !}
$$

different configurations for $2 m$ half-edges. 
Recall that the state space of the SESP Markov chain $\Omega$ consists of all possible multigraphs on $n$ labeled vertices and $2 m$ labeled half-edges. A multigraph $s \in \Omega$ is represented by a vector that contains for every vertex $v$ the set of adjacent half-edges $H(v)$ as defined in Section 1.1. We partition these states such that two graphs are in the same partition if and only if they exhibit the same degree sequence $d\left(v_{1}\right), \ldots, d\left(v_{n}\right)$. Let $\Omega^{\prime}$ be the state space defined by all degree sequences, and for a partitioning $\Omega^{\prime}$ of the state space $\Omega$, let $\phi(v): \Omega \rightarrow \Omega^{\prime}$ be the unique $x$ in $\Omega^{\prime}$ such that $v$ is an element of $x$. We will use the following lemma to prove that in the stationary distribution of SESP, all multigraphs of the same degree sequence appear with equal probability as in the configuration model. This means that we can use the configuration model to study $G_{n, a, \bar{k}}$.

Note that a degree distribution is often given as the number of vertices of a certain degree $N(1), N(2), \ldots, N(2 m)$ and not as the sequence of the degrees $d\left(v_{1}\right), \ldots, d\left(v_{n}\right)$. But obviously for a fixed $N(1), N(2), \ldots, N(2 m)$, every sequence of degrees (that matches $N(1), N(2), \ldots, N(2 m)$ ) appears with the same probability, since it is only a matter of relabeling the initial graph.

Lemma 3.I. If $\Omega^{\prime}$ is a partition of the ergodic Markov chain $\Omega, P$, $\pi$ that satisfies

$$
\forall_{x, y \in \Omega^{\prime}} \forall_{u, v \in y}: \sum_{s \in x} P(s, u)=\sum_{s \in x} P(s, v)
$$

and

$$
\forall_{x, y \in \Omega^{\prime}} \forall_{u, v \in x}: \sum_{s \in y} P(u, s)=\sum_{s \in y} P(v, s)
$$

then

$$
\forall_{v \in \Omega}: \pi(v)=\frac{\sum_{u \in \phi(v)} \pi(u)}{|\phi(v)|} .
$$

Proof. Because of ergodicity it is sufficient to show that for all $v \in \Omega$,

$$
\sum_{w \in \Omega} P(w, v) \frac{\sum_{u \in \phi(w)} \pi(u)}{|\phi(w)|}=\frac{\sum_{s \in \phi(v)} \pi(s)}{|\phi(v)|}
$$

is satisfied. First note that

$$
\begin{gathered}
\forall_{x \in \Omega^{\prime}} \forall_{u \in x} \forall_{v \in \Omega}: \sum_{w \in x} P(w, v) \stackrel{(3.8)}{=} \frac{1}{|\phi(v)|} \sum_{s \in \phi(v)} \sum_{w \in x} P(w, s) \\
\stackrel{(3.9)}{=} \frac{|x|}{|\phi(v)|} \sum_{s \in \phi(v)} P(u, s) .
\end{gathered}
$$


Hence for arbitrary $v \in \Omega$,

$$
\begin{aligned}
\sum_{w \in \Omega} P(w, v) \frac{\sum_{u \in \phi(w)} \pi(u)}{|\phi(w)|} & =\sum_{x \in \Omega^{\prime}} \frac{1}{|x|} \sum_{u \in x} \pi(u) \sum_{w \in x} P(w, v) \\
& \stackrel{(3.10)}{=} \frac{1}{|\phi(v)|} \sum_{x \in \Omega^{\prime}} \sum_{u \in x} \pi(u) \sum_{s \in \phi(v)} P(u, s) \\
& =\frac{1}{|\phi(v)|} \sum_{s \in \phi(v)} \sum_{w \in \Omega} P(u, s) \pi(u)=\frac{\sum_{s \in \phi(v)} \pi(s)}{|\phi(v)|}
\end{aligned}
$$

always holds.

We now use Lemma 3.1 to prove that for every degree sequence $s \in \Omega^{\prime}$ and every multigraph with labeled vertices and half-edges $G_{i} \in s$, the stationary distribution is $\pi\left(G_{i}\right)=\sum_{u \in s} \pi(u) /|s|$.

Proof of Theorem I.7. To apply Lemma 3.1 we need to show only that the SESP Markov chain satisfies (3.8) and (3.9). We first prove (3.8) for arbitrary degree sequences $x, y \in \Omega^{\prime}$.

We first assume that $x \neq y$. If there is a positive transition probability from a state $s$ in $x$ to a state $w$ in $y$, this means that there are one half-edge $e$ and two vertices $v$ and $v^{\prime}$ such that

$$
H_{s}(v)=H_{w}(v) \cup\{e\} \quad \text { and } \quad H_{w}\left(v^{\prime}\right)=H_{s}\left(v^{\prime}\right) \cup\{e\},
$$

and for all other vertices, $u \in V-\left\{v, v^{\prime}\right\} H_{s}(u)=H_{w}(u)$. Then a transition from $s$ to $w$ happens if and only if $e$ is selected and rewired to $v^{\prime}$. Thus, there are nonzero transition probabilities from $x$ to $y$ if and only if there is exactly one pair of vertices $v, v^{\prime}$ such that

$$
d_{x}(v)=d_{y}(v)+1 \quad \text { and } \quad d_{x}\left(v^{\prime}\right)=d_{y}\left(v^{\prime}\right)-1
$$

Then each multigraph $w \in y$ has exactly $d_{y}\left(v^{\prime}\right)$ different multigraphs $s \in x$ that have transition probability

$$
P(s, w)=\frac{1}{2 m} \frac{a \cdot d_{x}\left(v^{\prime}\right)+b}{n(a \bar{k}+b)},
$$

and hence (3.8) is satisfied.

If $x=y$, then for all $s, w \in x, P(s, w)>0$ implies that $s=w$, and therefore (3.8) is satisfied, because

$$
P(s, s)=P(w, w)=\sum_{v \in V} \frac{d_{x}(v)}{2 m} \frac{a \cdot d_{x}(v)+b}{n(a \bar{k}+b)} .
$$


The above observations can be applied analogically to show that (3.9) is also satisfied.

We observe that for a given degree sequence $d\left(v_{1}\right), d\left(v_{2}\right), \ldots, d\left(v_{n}\right)$ every labeling of the half-edges appears with the same probability. Choosing a labeling of the half-edges uniformly at random and connecting the two half-edges of each edge is equivalent to choosing a pairing of the half-edges uniformly at random out of all $(2 m-1)$ !! pairings.

\subsection{Properties of the Degree Sequence}

In [Molloy and Reed 95, Molloy and Reed 00] the authors defined the following natural properties for degree sequences of large graphs. An asymptotic degree sequence is a sequence of integer-valued functions $\mathbb{D}=d_{0}(n), d_{1}(n), \ldots$ such that

1. $d_{i}(n)=0$ for $i \geq n$,

2. $\sum_{i \geq 0} d_{i}(n)=n$.

An asymptotic degree sequence $\mathbb{D}$ is well behaved if:

1. $\mathbb{D}$ is smooth, which means that there exist constants $\lambda_{i}$ such that

$$
\lim _{n \rightarrow \infty} \frac{d_{i}(n)}{n}=\lambda_{i}
$$

where $n$ denotes the total number of vertices and $d_{i}(n)$ the number of vertices of degree $i$.

2. For all $\varepsilon>0$ there exists $n_{0}$ such that for all $n>n_{0}$ and for all $i \geq 0$,

$$
\left|\frac{i(i-2) d_{i}(n)}{n}-i(i-2) \lambda_{i}\right|<\varepsilon .
$$

3.

$$
L(\mathbb{D})=\lim _{n \rightarrow \infty} \sum_{i \geq 1} \frac{i(i-2) d_{i}(n)}{n}
$$

exists, and the sum approaches the limit uniformly, i.e., if $L(\mathbb{D})$ is finite, then for all $\varepsilon>0$, there exist $i^{*}, n_{0}$ such that for all $n>n_{0}$,

$$
\left|\sum_{i=1}^{i^{*}} \frac{i(i-2) d_{i}(n)}{n}-L(\mathbb{D})\right|<\varepsilon,
$$


or if $L(\mathbb{D})$ is infinite, then for all $T>0$, there exist $i^{*}, n_{0}$ such that for all $n>n_{0}$,

$$
\sum_{i=1}^{i^{*}} \frac{i(i-2) d_{i}(n)}{n}>T
$$

An asymptotic degree sequence $\mathbb{D}$ is sparse if

$$
\sum_{i \geq 0} \frac{i d_{i}(n)}{n}=K+o(1)
$$

for some constant $K$.

Lemma 3.2. For constant $\bar{k}$, the degree sequence of $G_{n, a, \bar{k}}$ is whp a well-behaved and sparse asymptotic degree sequence.

Proof. By Theorem 1.7, we can fix a graph $G \sim G_{n, a, \bar{k}}$ by first exposing its degree sequence $N(0), \ldots, N(2 m)$ and then choosing a random multigraph of that degree sequence by employing the configuration model. We show that the degree sequence is well behaved whenever the maximum degree is at most $k_{\max }:=\left(\frac{3}{\log c_{2}}\right) \log m$ and

$$
\forall_{0 \leq k \leq k_{\text {max }}}: n \cdot P(k)-\sqrt{n \log n} \leq N(k) \leq n \cdot P(k)+\sqrt{n \log n}
$$

holds. Note that $n \cdot P(k)=\mathbb{E}[N(k)]$, and the two events are therefore by Corollary 1.6 and Theorem 1.5 satisfied with probability at least

$$
1-\left(\frac{3}{\log c_{2}}\right) \log m \cdot 2 n^{-1 /\left(4 c_{1}\right)}-o(1)=1-o(1) .
$$

It remains to show that the degree sequence $d_{0}(n), \ldots, d_{2 m}(n)=$ $N(0), \ldots, N(2 m)$ is well behaved if (3.12) is satisfied and the maximum degree is at most $k_{\max }$.

Clearly, $\sum_{i \geq 0} d_{i}(n)=n$ and $d_{i}(n)=0$ for $i>n-1$. Let $\lambda_{i}:=P(i)$ for $i \leq$ $k_{\max }:=\left(\frac{3}{\log c_{2}}\right) \log m$ and $\lambda_{i}=0$ for larger $i$. Because of (3.12),

$$
\frac{d_{i}(n)}{n}=\lambda_{i} \pm O\left(\frac{\sqrt{n \log n}}{n}\right)
$$

and therefore

$$
\lim _{n \rightarrow \infty} \frac{d_{i}(n)}{n}=\lambda_{i}
$$


For $i>k_{\max }$, we have that

$$
\left|\frac{i(i-2) d_{i}(n)}{n}-i(i-2) \lambda_{i}\right|=0,
$$

while if $i \leq k_{\max }$, then

$$
\left|\frac{i(i-2) d_{i}(n)}{n}-i(i-2) \lambda_{i}\right|=\left|i(i-2) \frac{O(\sqrt{n \log n})}{n}\right|=o(1)
$$

where the last equality follows from $i \leq k_{\mathrm{max}}$, and therefore the second condition for a well-behaved degree sequence is satisfied. For the third condition, we inspect $L(\mathbb{D})$ :

$$
\begin{aligned}
L(\mathbb{D}) & =\lim _{n \rightarrow \infty}\left(\sum_{i \geq 1} i(i-2) \lambda_{i}+\sum_{i=1}^{k_{\max }} i(i-2) \frac{O(\sqrt{n \log n)}}{n}\right) \\
& =\lim _{n \rightarrow \infty}\left(\sum_{i \geq 1} i(i-2) \lambda_{i}+o(1)\right)=\sum_{i=1}^{\infty} i(i-2) \lambda_{i} .
\end{aligned}
$$

This means that the limit exists, and because

$$
\lambda_{i}=O\left(\left(1+\frac{b}{a \bar{k}}\right)^{-i}\right),
$$

the sum

$$
\sum_{i \geq 1}^{\infty} i(i-2) \lambda_{i}
$$

converges. Hence for all $\epsilon>0$ there exists a constant $i^{*}$ such that

$$
\sum_{i=i^{*}+1}^{\infty} i(i-2) \lambda_{i}<\epsilon,
$$

and therefore for some $n_{0}$ and all $n>n_{0}$,

$$
\begin{aligned}
\left|\sum_{i=1}^{i^{*}} \frac{i(i-2) d_{i}(n)}{n}-L(\mathbb{D})\right| & \stackrel{(3.13)}{=}\left|\sum_{i=1}^{i^{*}} i(i-2) \frac{d_{i}(n)}{n}-\sum_{i=1}^{\infty} i(i-2) \lambda_{i}\right| \\
& =\left|\sum_{i=1}^{i^{*}} i(i-2)\left(d_{i}(n) / n-\lambda_{i}\right)-\sum_{i=i^{*}+1}^{\infty} i(i-2) \lambda_{i}\right| \\
& =\left|o(1)-\sum_{i=i^{*}+1}^{n} i(i-2) \lambda_{i}\right|<\epsilon .
\end{aligned}
$$


Note that [Fernholz and Ramachandran 07] defines a similar set of properties, for which the authors proved that the diameter of random graphs satisfying those properties is $c \ln n+o(\ln n)$. By combining their results and our framework, it might be possible to prove that the diameter of $G_{n, a, \bar{k}}$ is $O(\ln n)$.

\section{Applications}

In this section we demonstrate how our main results can be used to study properties of $G_{n, a, \bar{k}}$. We can use the configuration model (Theorem 1.7) and the degree distribution (Theorem 1.5 and Corollary 1.6) to calculate the expectation of random variables in $G_{n, a, \bar{k}}$. As long as those random variables can be formulated as 1-Lipschitz functions, we can then apply Theorem 1.4 to derive bounds on their concentration. The weakness of SESP is that it is not restricted to simple graphs, which means that it can produce graphs with self-loops. In [Dorogovtsev and Mendes 03], the authors indicated that the number of self-loops is small. In Section 4.1 we give a short rigorous proof of this fact. In Section 4.2 we use a result that was derived for the configuration model to study the emergence of the giant component in $G_{n, a, \bar{k}}$. This exemplifies how known results can be transferred from the configuration model to $G_{n, a, \bar{k}}$.

\section{I. Self-Loops}

We now use the configuration model to derive the number of self-loops in $G_{n, a, \bar{k}}$. Note that in the configuration model, the probability that two specific half-edges are connected is exactly

$$
\frac{(2 m-3) ! !}{(2 m-1) ! !}=\frac{1}{2 m-1}
$$

The expected number of self-loops attached to a vertex of degree $k$ is $\left(\begin{array}{l}k \\ 2\end{array}\right) \frac{1}{2 m-1}$, since there are $\left(\begin{array}{l}k \\ 2\end{array}\right)$ pairs of half-edges that are connected with probability $\frac{1}{2 m-1}$. We can calculate the expected number of self-loops on an arbitrary vertex by summing over all possible degrees $k$ :

$$
\sum_{k} p(k)\left(\begin{array}{l}
k \\
2
\end{array}\right) \frac{1}{2 m-1}=\frac{\left\langle\left(\begin{array}{l}
k \\
2
\end{array}\right)\right\rangle}{2 m-1} .
$$

Therefore, the expected number of self-loops in the graph is $\frac{n \cdot\left\langle\left(\begin{array}{c}k \\ 2\end{array}\right)\right\rangle}{2 m-1}$. In $G_{n, a, \bar{k}}$,

$$
\left\langle\left(\begin{array}{l}
k \\
2
\end{array}\right)\right\rangle=O\left(\sum_{k}^{2 m} k^{1+\frac{b}{a}}\left(1+\frac{b}{a \bar{k}}\right)^{-k}\right)=O(1)
$$


because the infinite sum

$$
\sum_{k}^{\infty} k^{1+\frac{b}{a}}\left(1+\frac{b}{a \bar{k}}\right)^{-k}
$$

converges.

\subsection{The Giant Component}

In 1995, Molloy and Reed [Molloy and Reed 95, Molloy and Reed 00] discovered the critical point for random graphs of a given asymptotic degree sequence. For a sequence of nonnegative real numbers $\lambda_{0}, \lambda_{1}, \ldots$, they showed that a random graph that has approximately $\lambda_{i} n$ vertices of degree $i$ has a.a.s. a giant component if $\sum i(i-2) \lambda_{i}>0$, while if $\sum i(i-2) \lambda_{i}<0$, then a.a.s. all components are small.

Molloy and Reed introduced two lemmas on configurations to prove their results on random simple graphs. We merge those two lemmas, [Molloy and Reed 95, Lemmas 3 and 7], into the following one.

Lemma 4.I. For a well-behaved and sparse asymptotic degree sequence $\mathbb{D}$, let $Q(\mathbb{D}):=\sum_{i \geq 0} i(i-1) \lambda_{i}, \quad \sum_{i \geq 0} i d_{i}(n) / n=K+o(1), \quad v=-Q(\mathbb{D}) / K, \quad R=$ $150 / v^{2}$, and let $F$ be a random configuration with $n$ vertices and degree sequence $\mathbb{D}$. If $Q(\mathbb{D})<0$ and if for some function $0 \leq \omega(n) \leq n^{1 / 8-\epsilon}, F$ has no vertices of degree greater than $\omega(n)$, then $F$ a.a.s. has no component with more than $\alpha=\left\lceil R \omega(n)^{2} \log n\right\rceil$ vertices. If $Q(\mathbb{D})>0$, then there exist constants $\zeta_{1}, \zeta_{2}>0$ dependent on $\mathbb{D}$ such that a.a.s. $F$ has a component with at least $\zeta_{1} n$ vertices and $\zeta_{2} n$ cycles.

In Section 3.2 we showed that $G \sim G_{n, a}, \bar{k}$ a.a.s. meets the conditions of Lemma 4.1 with $\lambda_{i}:=P(i)$ for $i \leq k_{\max }:=\left(\frac{3}{\log c_{2}}\right) \log m$, and $P(i)=0$ otherwise. Therefore, a graph $G \sim G_{n, a, \bar{k}}$ has a.a.s. a giant component if $Q(\mathbb{D})>0$, while if $Q(\mathbb{D})<0$, then almost surely all components have size $o(n)$. This is formulated in the following lemma.

Lemma 4.2. Let $Q=\sum_{k=1}^{k_{\max }} k(k-2) P(k)$, where

$$
P(k)=\prod_{i=1}^{k} \frac{f(i-1) \cdot(2 m-i+1)}{i \cdot(n\langle f\rangle-f(i))} / \sum_{j=0}^{2 m} \prod_{i=1}^{j} \frac{f(i-1) \cdot(2 m-i+1)}{i \cdot(n\langle f\rangle-f(i))}
$$

and

$$
k_{\max }:=\left(\frac{3}{\log c_{2}}\right) \log m .
$$


Then for $Q>0, G \sim G_{n, a, \bar{k}}$ has a.a.s. a component of $\Theta(n)$ vertices, while for $Q<0$, there is a.a.s. no component with more than $O\left(\log ^{2} n\right)$ vertices.

Lemma 4.2 characterizes for which parameters $a$ and $\bar{k}$ the giant component in $G_{n, a, \bar{k}}$ emerges. The goal is to determine a simpler function of $a$ and $\bar{k}$ that decides whether $G_{n, a, \bar{k}}$ has a giant component. We believe that $Q<0$ if $\bar{k}<b$ and that $Q>0$ if $\bar{k}>b$.

Conjecture 4.3. Let $G \sim G_{n, a, \bar{k}}$. Then for $\bar{k}>b, G$ has a.a.s. a component of size $\Theta(n)$, and for $\bar{k}<b$, there is a.a.s. no component of size larger than $O\left(\log ^{2} n\right)$.

The cases $a=b$ and $b=1$ as well as numerical calculations confirm this conjecture. We now show that the conjecture is true for $a=b$ and $b=1$.

In (3.3) we derived that the degree distribution for $b=1$ and $a=0$ is

$$
P(k)=\left(\begin{array}{c}
2 m \\
k
\end{array}\right)\left(\frac{1}{n}\right)^{k}\left(\frac{n-1}{n}\right)^{2 m-k}
$$

Let $c$ be a constant. Then for $m=c(n-1)$ it follows that

$$
\begin{aligned}
Q & =\left(\frac{n-1}{n}\right)^{2 c(n-1)} \sum_{i=1}^{k_{\mathrm{max}}} i(i-2)\left(\begin{array}{c}
2 c(n-1) \\
i
\end{array}\right)(n-1)^{-i} \\
& =\left(\frac{n-1}{n}\right)^{2 c(n-1)}\left(-2 c+\sum_{i=3}^{k_{\mathrm{max}}} i(i-2)\left(\begin{array}{c}
2 c(n-1) \\
i
\end{array}\right)(n-1)^{-i}\right) .
\end{aligned}
$$

For $c<1 / 2$, this means that $Q<0$, because

$$
-2 c+\sum_{i=3}^{k_{\max }} i(i-2)\left(\begin{array}{c}
2 c(n-1) \\
i
\end{array}\right)(n-1)^{-i} \leq-2 c+\sum_{i \geq 3} i(i-2) \frac{(2 c)^{i}}{i !}<0
$$

while $Q>0$ for $c>1 / 2$, since

$$
-2 c+\sum_{i=3}^{k_{\mathrm{max}}} i(i-2)\left(\begin{array}{c}
2 c(n-1) \\
i
\end{array}\right)(n-1)^{-i}=-2 c+\sum_{i=3}^{k_{\mathrm{max}}} i(i-2) \frac{(2 c)^{i}}{i !}(1-o(1))>0
$$

for $n$ large enough. 
Another case for which we derive a threshold for the giant component is $a=b=1 / 2$. Then $Q$ in Lemma 4.2 simplifies to

$$
\begin{aligned}
Q & =\frac{1}{c} \sum_{i=1}^{k_{\max }} i(i-2) \prod_{k=1}^{i} \frac{(a(k-1)+b)(2 m-k+1)}{k(a(2 m-k)+b(n-1))} \\
& =\frac{1}{c} \frac{b 2 m}{a(2 m-1)+b(n-1)}\left(-1+\sum_{i=3}^{k_{\max }} \frac{(i-2)}{(i-1) !} \prod_{k=2}^{i} \frac{\left(k-1+\frac{b}{a}\right)\left(\bar{k}-\frac{k-1}{n}\right)}{\bar{k}+\frac{b(n-1)}{a n}-\frac{k}{n}}\right),
\end{aligned}
$$

where $c$ is the normalization term of $P(k)$. Therefore $Q \stackrel{(<)}{>} 0$ if and only if

$$
Q^{\prime}:=\sum_{i=3}^{k_{\max }} i(i-2) \prod_{k=2}^{i} \frac{\bar{k}-\frac{k-1}{n}}{\bar{k}+1-\frac{k+1}{n}} \stackrel{(<)}{>} 1 \text {. }
$$

For $\bar{k}=1 / 2-\varepsilon$,

$$
Q^{\prime}=\sum_{i=3}^{k_{\mathrm{max}}} i(i-2) \prod_{k=2}^{i}\left(1-\frac{1-\frac{2}{n}}{\frac{3}{2}-\varepsilon-\frac{k+1}{n}}\right)<\sum_{i \geq 3} i(i-2)\left(\frac{1}{3}\right)^{i-1}=1,
$$

because

$$
f(z):=1-\frac{1}{(1-z)^{2}}+\frac{2 z}{(1-z)^{3}}=\sum_{i \geq 0}(i+2) i z^{i+1}
$$

and $f(1 / 3)=1$. For $\bar{k}=1 / 2+\varepsilon$,

$$
Q^{\prime}=\sum_{i=3}^{k_{\text {max }}} i(i-2) \prod_{k=2}^{i}\left(1-\frac{1-\frac{2}{n}}{\frac{3}{2}+\varepsilon-\frac{k+1}{n}}\right) \stackrel{i+1<\varepsilon n / 2}{>} \sum_{i=3}^{k_{\max }} i(i-2)\left(\frac{1+\varepsilon}{3+\varepsilon}\right)^{i-1}>1
$$

for $n$ large enough, since $f\left(\frac{1+\varepsilon}{3+\varepsilon}\right)=1+\varepsilon\left(\frac{3+\varepsilon}{2}\right)^{2}$.

\section{Conclusion}

In this paper we have described a new way of looking at nongrowing networks. By considering the stationary distribution of SESP, we defined a new random graph model $G_{n, a, \bar{k}}$ and developed a framework to study its properties. We underlined the relevance of this new approach by showing that the model has optimal mixing time and therefore reaches the stationary phase in $O(m \log m)$ steps. This means that a real-world network (for which the model fits) is with high probability already in the stationary state.

A different approach was chosen in [Evans and Plato 07], where the authors solved a mean-field equation for the degree distribution after step $t$ of the process. 
If we look at their solution for $t=\infty$, we get

$$
P(k) \approx k^{-1+\frac{p_{r}}{p_{p}} \bar{k}} \cdot p_{p}^{k}
$$

for their preference function $f(k)=p_{p} \frac{k}{2 m}+p_{r} \frac{1}{n}$, where $p_{p}+p_{r}=1$.

Note that for

$$
p_{p}=\frac{a}{a+\frac{b}{\bar{k}}} \quad \text { and } \quad p_{r}=\frac{b}{a \bar{k}+b}
$$

the two models are equivalent. By substituting our $p_{p}$ and $p_{r}$ into (5.1), yielding

$$
P(k) \approx k^{-1+\left(\frac{b}{a \bar{k}+b}\right) \bar{k} /\left(\frac{a}{a+b / \bar{k}}\right)} \cdot\left(\frac{a}{a+b / \bar{k}}\right)^{k}=\frac{1}{\left(1+\frac{b}{a \bar{k}}\right)^{k}} \cdot k^{-1+b / a},
$$

we see that our solution confirms their result in the limit.

We believe that our framework can be used similarly to the methods known from traditional random graph models such as the Erdös-Rényi model and the configuration model. The discussion of the giant component in Section 4.2 illustrates the building blocks of the framework. The equivalence to the configuration model (Theorem 1.7) and the concentration of 1-Lipschitz functions (Theorem 1.4) allows us to apply a result of [Molloy and Reed 95]. We therefore used the degree distribution (derived in Section 3) to analyze the value of $\sum k(k-2) P(k)$.

A natural question for future research is to develop an analogical theory for simple graphs. The natural attempt to ignore step 4 of Process 1.1 whenever it would create a self-loop or a multiedge leads to considerable difficulties in the process analysis (see [Hruz et al. 08]). To overcome these obstacles, we see basically two possibilities. Either one studies multigraphs and uses the fact, which we prove in the present paper, that there is only a very small number of self-loops (and analogically multiple edges), or one tries to study new meaningful processes that create scale-free distributions.

\section{Asymptotic Degree Sequences}

The following technical lemma is used in Section 3.

Lemma 6.I. For $n=2 m / \bar{k}$ and $0 \leq k \leq 2 m$,

$$
\frac{\Gamma\left(k+\frac{b}{a}\right)}{\Gamma(k+1)} \frac{\Gamma(2 m+1)}{\Gamma(2 m-k+1)} \frac{\Gamma\left(2 m-k+\frac{b}{a}(n-1)\right)}{\Gamma\left(2 m+\frac{b}{a}(n-1)\right)}=O\left(k^{-1+\frac{b}{a}} c_{2}^{-k}\right),
$$


and for $0 \leq k \leq\left(\frac{2+\varepsilon}{\log c_{2}}\right) \log m$,

$$
\frac{\Gamma\left(k+\frac{b}{a}\right)}{\Gamma(k+1)} \frac{\Gamma(2 m+1)}{\Gamma(2 m-k+1)} \frac{\Gamma\left(2 m-k+\frac{b}{a}(n-1)\right)}{\Gamma\left(2 m+\frac{b}{a}(n-1)\right)}=\Theta\left(k^{-1+\frac{b}{a}} c_{2}^{-k}\right),
$$

where $c_{2}:=1+\frac{b}{a \bar{k}}$.

Proof. We use Stirling's approximation

$$
\Gamma(z)=\sqrt{2 \pi z}\left(\frac{z}{e}\right)^{z}\left(\left(1+O\left(\frac{1}{z}\right)\right)\right.
$$

to obtain

$$
\frac{\Gamma\left(k+\frac{b}{a}\right)}{\Gamma(k+1)}=\sqrt{\frac{k+\frac{b}{a}}{k+1}} e^{1-\frac{b}{a}}\left(\frac{k+\frac{b}{a}}{k+1}\right)^{k} \frac{\left(k+\frac{b}{a}\right)^{\frac{b}{a}}}{(k+1)} \Theta(1)=\Theta\left(k^{-1+\frac{b}{a}}\right),
$$

where we have used $\left(1+\frac{\frac{b}{a}-1}{k+1}\right)^{k}=\Theta(1)$, which follows from well-known features of the exponential function. For $n=2 m / \bar{k}$ we apply again the above Stirling's approximation to the remaining terms:

$$
\begin{aligned}
& \frac{\Gamma(2 m+1)}{\Gamma(2 m-k+1)} \frac{\Gamma\left(2 m-k+\frac{b}{a}(n-1)\right)}{\Gamma\left(2 m+\frac{b}{a}(n-1)\right)} \\
&=\sqrt{\frac{(2 m+1)\left(2 m-k+\frac{b}{a}(n-1)\right)}{(2 m-k+1)\left(2 m+\frac{b}{a}(n-1)\right)}}\left(\frac{2 m+1}{2 m-k+1}\right)^{2 m-k+1}(2 m+1)^{k} \\
& \cdot\left(\frac{2 m-k+\frac{b}{a}(n-1)}{2 m+\frac{b}{a}(n-1)}\right)^{2 m-k+\frac{b}{a}(n-1)} \frac{1}{\left(2 m+\frac{b}{a}(n-1)\right)^{k}} \cdot \Theta(1) \\
&=\left(\frac{2 m+1}{2 m-k+1}\right)^{2 m-k+1+\frac{1}{2}}(2 m+1)^{k}\left(\frac{2 m-k+\frac{b}{a}(n-1)}{2 m+\frac{b}{a}(n-1)}\right)^{2 m-k+\frac{b}{a}(n-1)+\frac{1}{2}} \\
& \cdot \frac{1}{\left(2 m+\frac{b}{a}(n-1)\right)^{k}} \Theta(1) \\
&= \Theta\left(\left(\frac{2 m+1}{2 m-k+1}\right)^{2 m-k+1+\frac{1}{2}}\left(\frac{2 m c_{2}-k}{2 m c_{2}}\right)^{2 m c_{2}-k+\frac{1}{2}} c_{2}^{-k}\right)
\end{aligned}
$$

where $c_{2}:=1+\frac{b}{a \bar{k}}$. We furthermore observe that

$$
\phi(k):=\left(\frac{2 m+1}{2 m-k+1}\right)^{2 m-k+1+\frac{1}{2}}\left(\frac{2 m c_{2}-k}{2 m c_{2}}\right)^{2 m c_{2}-k+\frac{1}{2}}
$$


is monotonically decreasing for $k \in\{0, \ldots, 2 m\}$ and $m$ large enough. Since $\phi(0)=1$, we conclude that

$$
\Theta\left(\left(\frac{2 m+1}{2 m-k+1}\right)^{2 m-k+1+\frac{1}{2}}\left(\frac{2 m c_{2}-k}{2 m c_{2}}\right)^{2 m c_{2}-k+\frac{1}{2}} c_{2}^{-k}\right)=O\left(c_{2}^{-k}\right) .
$$

For $0 \leq k\left(\frac{2+\varepsilon}{\log c_{2}}\right) \log m$, we have

$$
\begin{aligned}
\phi(k) & =\left(1-\frac{k}{2 m+1}\right)^{-(2 m+1)+k-1 / 2}\left(1-\frac{k}{2 m c_{2}}\right)^{2 m c_{2}-k+1 / 2} \\
& =(1+o(1)) e^{-\frac{k^{2}-k / 2}{2 m+1}+\frac{k^{2}-k / 2}{2 m c_{2}}} \\
& \geq(1+o(1)) e^{-\frac{k}{2 m}\left(1-\frac{1}{c_{2}}\right)(k-1 / 2)} \\
& \geq(1+o(1)) m^{-\frac{\left(\frac{2+\varepsilon}{\log c_{2}}\right)\left(1-\frac{1}{c_{2}}\right)(k-1 / 2)}{2 m}}=\Theta(1) .
\end{aligned}
$$

Acknowledgments The authors would like to thank Peter Widmayer for stimulating discussions and continuing support of this project. We would also like to thank the anonymous reviewers for suggestions that helped to improve the text.

\section{References}

[Aldous 83] David Aldous. "Random Walks on Finite Groups and rapidly mixing Markov chains." Séminaire de Probabilités XVII 1981/82 (1983), 243-297.

[Barabási and Albert 99] A.-L. Barabási and R. Albert. Emergence of Scaling in Random Networks. Science 286 (1999), 509-512.

[Bollobás 80] Béla Bollobás. "A Probabilistic Proof of an Asymptotic Formula for the Number of Labelled Regular Graphs." Europ. J. Combin. 1 (1980), 311-316.

[Bollobás et al. 01] Béla Bollobás, Oliver Riordan, Joel Spencer, and Gábor Tusnády. "The Degree Sequence of a Scale-Free Random Graph Process." Random Structures and Algorithms 18 (2001), 279-290.

[Cami and Deo 07] A. Cami and N. Deo. "Techniques for Analyzing Dynamic Random Graph Models of Web-like Networks: An Overview." Networks 51:4 (2007), 211-255.

[Dorogovtsev and Mendes 03] S. N. Dorogovtsev and J. F. F. Mendes. Evolution of Networks. Oxford: Oxford University Press, 2003.

[Dudley 02] R. M. Dudley. Real Analysis and Probability. Cambridge, UK: Cambridge University Press, 2002.

[Dyer et al. 00] Martin E. Dyer, Leslie Ann Goldberg, Catherine S. Greenhill, Mark Jerrum, and Michael Mitzenmacher. "An Extension of Path Coupling and Its Application to the Glauber Dynamics for Graph Colourings" (extended abstract). In Symposium on Discrete Algorithms, pp. 616-624, 2000. 
[Evans and Plato 07] T. S. Evans and A. D. K. Plato. "Exact Solution for the Time Evolution of Network Rewiring Models." Physical Review E 75(056101), 2007.

[Fernholz and Ramachandran 07] Daniel Fernholz and Vijaya Ramachandran. "The Diameter of Sparse Random Graphs." Random Struct. Algorithms 31:4 (2007), 482-516.

[Hruz et al. 08] T. Hruz, M. Natora, and M. Agrawal. "Higher-Order Distributions and Nongrowing Complex Networks without Multiple Connections." Physical Review E 77 (2008), 046101.

[Janson et al. 00] Svante Janson, Tomasz Luczak, and Andrzej Rucinski. Random Graphs. New York: Wiley-Interscience, 2000.

[Levin et al. 09] D. A. Levin, Y. Peres, and E. L. Wilmer. Markov Chains and Mixing Time. Providence: American Mathematical Society, 2009.

[Luczak 08] Malwina J. Luczak. "Concentration of Measure and Mixing for Markov Chains" DMTCS Proceedings (2008), 95-120.

[Mitzenmacher 06] Michael Mitzenmacher. "The Future of Power Law Research," editorial. Internet Mathematics 2 (2006), 525-534.

[Molloy and Reed 95] Michael Molloy and Bruce Reed. "A Critical Point for Random Graphs with a Given Degree Sequence" Random Structures and Algorithms (1995), 161-180.

[Molloy and Reed 00] Michael Molloy and Bruce Reed. "The Size of the Giant Component of a Random Graph with a Given Degree Sequence." Combinatorics, Probability and Computing 7:3 (2000), 295-305.

[Park et al. 05] Kwangho Park, Ying-Cheng Lai, and Nong Ye. "Self-Organized ScaleFree Networks." Physical Review E 72 (2005), 026131.

[Watts and Strogatz 98] D. J. Watts and S. H. Strogatz. "Collective Dynamics of SmallWorld Networks." Nature 393 (1998), 440-442.

Tomas Hruz, Institute of Theoretical Computer Science, ETH Zurich, 8092 Zurich, Switzerland (tomas.hruz@inf.ethz.ch)

Ueli Peter, Institute of Theoretical Computer Science, ETH Zurich, 8092 Zurich, Switzerland (upeter@ethz.ch)

Received July 19, 2010; accepted October 1, 2010. 